\title{
Subjective Age and Cognitive Functioning: A 10-Year Prospective Study
}

\author{
Yannick Stephan, Ph.D., Joban Caudroit, Ph.D., Alban Jaconelli, M.Sc., \\ Antonio Terracciano, Ph.D.
}

\begin{abstract}
Objectives: The present study examines whether subjective age (i.e., bow old or young individuals feel) is associated with cognitive functioning and tests potential mediators of this association. Design: Data from the two waves of measurement of the Midlife in the United States survey were used, with assessments conducted at the first wave in 1994-1995 and at the second wave in 2004-2006. Participants: A total of 1,352 men and women aged from 50 to 75 years at baseline (M: 59.32; SD: 6.72). Measurements: Subjective age, body mass index (BMI), physical activity, and the covariates sex, age, education, marital status, and disease burden were assessed at baseline to predict episodic memory and executive function measured 10 years later. Results: Multiple regression analysis revealed that a younger subjective age at baseline was prospectively associated with better episodic memory and executive function. Bootstrap analysis indicated that the association of subjective age with episodic memory and executive function was partially mediated by BMI and frequency of physical activity respectively. Conclusion: Even after accounting for chronological age and other risk factors for cognitive decline, such as disease burden and sedentary lifestyle, the subjective experience of aging predicts cognitive functioning in old age. (Am J Geriatr Psychiatry 2014; 22:1180-1187)
\end{abstract}

Key Words: Subjective age, episodic memory, executive function, prospective study, BMI, physical activity

C ognitive functions, such as memory and executive functions, are crucial for older individuals' abilities to conduct a variety of activities ranging from self care (instrumental activities of daily living) to more challenging tasks. ${ }^{1,2}$ Thus, preserved cognitive functioning is related to higher health-related quality of life among older adults. ${ }^{3}$ Indeed, deficits in memory and executive function are associated with increased risk of developing Alzheimer disease dementia. 4 Therefore, it is important to identify factors associated with cognitive functioning among older adults. Research on such factors can suggest

Received December 21, 2012; revised March 7, 2013; accepted March 8, 2013. From EA 4556 Epsylon, Department of Sport Sciences, Psychology and Medicine, University of Montpellier and St-Etienne (YS), Montpellier, France; CRIS EA 647, Department of Sport Sciences, University of Lyon 1 (JC), Lyon, France; EA 3742, Department of Sport Sciences, University of Grenoble (AJ), Grenoble, France; and Department of Geriatrics, College of Medicine, Florida State University (AT), Tallahassee, FL. Send correspondence and reprint requests to Yannick Stephan, Ph.D., EA 4556 EPSYLON, Laboratory Dynamic of Human Abilities and Health Behaviors, Department of Sport Sciences, Psychology and Medicine, University of Montpellier and St-Etienne, 4, Boulevard Henry IV, 34000 Montpellier, France. e-mail: yannick. stephan@univ-montp1.fr

(C) 2014 American Association for Geriatric Psychiatry

http:/ /dx.doi.org/10.1016/j.jagp.2013.03.007 
mechanisms that regulate the rate of cognitive decline. In addition, a refined identification of predictors of cognitive functions is needed to define segments of the population at greater risk and to target interventions. Furthermore, psychological factors associated with cognitive deficits may represent prodromal symptoms, and as such could be useful as a diagnostic marker.

Chronological age is the basic dimension along which cognitive functions are described and investigated among older adults. More specifically, research consistently finds increasing age as the main predictor of cognitive decline in normal aging. ${ }^{6}$ Age is also the most powerful predictor of incident dementia. ${ }^{7}$ Although chronological age is used as a key indicator of developmental changes and is considered as a risk factor for decrements in cognition, however, alternative ways of measuring developmental time could provide valuable insights about the mechanisms involved in older adults' cognitive functioning. ${ }^{8,9}$ In this vein, studies have found that biological age, reflecting the functioning of critical physiological systems and processes of an individual relative to his or her own life span, contributes to cognitive functioning, independent of chronological age. ${ }^{8,9}$ These findings are consistent with the view that older adults' cognition is influenced by a range of factors that operate along the age continuum. ${ }^{10-14}$

A growing body of research suggests that aging is also a subjective experience. ${ }^{15}$ There is an increasing discrepancy between subjective age and chronological age as people grow older, and the majority of older adults feel younger than they actually are despite age-related changes. ${ }^{15-17}$ Subjective age predicts important outcomes, including well-being, ${ }^{18}$ physical functioning, ${ }^{19}$ self-rated health, ${ }^{18}$ and longevity. ${ }^{20}$ In most of these studies, a younger subjective age contributes to more favorable psychological and health-related outcomes, independent of chronological age, and beyond other demographic factors (e.g., education, sex, marital status) and health variables (e.g., disease burden). ${ }^{18-21}$ To date, no research on the correlates of older adults' cognitive functioning have considered the potential contribution of subjective age.

There are reasons to expect that subjective age may be related to cognition in old age, given that it has been related to psychosocial, behavioral, and healthrelated processes that influence the rate of cognitive decline. Prior research has found a positive relationship between a younger subjective age and memory self-efficacy (the belief in the effectiveness of one's memory function, ${ }^{18,22}$ which contributes to the maintenance of memory performance with advancing $\operatorname{age}^{23}$ ). Health and lifestyle patterns are another argument in favor of a subjective age-cognition relationship. Individuals who feel younger than their age are generally healthier and less likely to suffer from chronic conditions, such as hypertension and diabetes. ${ }^{24}$ These individuals with a younger subjective age are more likely to engage in health-promoting behaviors, such as physical activity. ${ }^{25}$ Individuals with more active lifestyles perform better on cognitive testing, including memory and executive functioning, ${ }^{26,27}$ and are found to be at reduced risk of dementia. ${ }^{27}$ Therefore, an active lifestyle can be hypothesized to be a potential mediator of the association between subjective age and cognition in old age. Because of the association with a sedentary lifestyle and worse health status, including risk for cognitive impairment, ${ }^{28}$ obesity is another potential mediator of the association between subjective age and cognitive functioning. Therefore, it is likely that a younger subjective age may be related to better cognitive functioning due to its association with a reduced risk of obesity.

Based upon data from the two waves of the Midlife in the United States longitudinal survey (MIDUS), ${ }^{29}$ the main purpose of the present study is to examine whether subjective age assessed at baseline is prospectively associated with cognitive function 10 years later, assessed through measures of episodic memory and executive function. Based on prior research, ${ }^{18,25}$ it was hypothesized that a younger subjective age is associated with better cognitive performance among older adults. A complementary goal was to assess the mediation of this relationship by lifestyle factors (i.e., physical activity) and other known risk factors (i.e., body mass index [BMI]).

\section{METHOD}

\section{Study Sample}

Data were drawn from the two waves of the Midlife in the United States study. ${ }^{30,31}$ Wave 1 data were collected in 1994-1995 and included a sample 
of 7,108 adults aged $20-79$ years. Wave 2 data were collected in 2004-2005 and consisted of 4,963 adults aged $28-84$ years, which is approximately $70 \%$ of the original sample, adjusted for mortality. ${ }^{31}$ We restricted analysis to participants who were 50 years old and over at baseline. The response rate was around $61 \%$. As is typically found, those who participated at the second wave of the MIDUS showed positive selection on most variables. ${ }^{31}$ The longitudinal sample was younger $(\mathrm{t}(2750)=-4.68$, $\mathrm{p}<0.001)$, more educated $(\mathrm{t}(2799)=7.25, \mathrm{p}<0.001)$, had a lower disease burden $(\mathrm{t}(2548)=-5.22$, $\mathrm{p}<0.001)$, was more physically active $(\mathrm{t}(2518)=7.65$, $\mathrm{p}<0.001)$, and felt slightly younger $(\mathrm{t}(2454)=2.27$, $\mathrm{p}<0.05)$, than the drop-out sample. After having excluded 166 participants with a history of stroke, Parkinson disease, or other neurologic disorders, the final analyzed sample consisted of 1,368 individuals who presented complete data on all measures. They were aged from 50 to 75 years at baseline (mean age: 59.35; SD: 6.73), 55\% were women, $76 \%$ were currently married or living with someone, and $31 \%$ graduated from high school.

\section{Measures}

In line with previous studies, ${ }^{16,17,20,21,32}$ subjective age was assessed at baseline by asking participants to specify, in years, how old they felt most of the time. Participants' felt age was subtracted from their chronological age, and these difference scores were divided by chronological age. ${ }^{17,19,33}$ A discrepancy score was obtained, reflecting how old or young individuals feel relative to their chronological age. Furthermore, this score takes into account that a discrepancy between felt age and actual age might have different meanings depending on the age of a person, and thus is applicable and comparable across different age groups. ${ }^{17,19,33}$ A positive value denotes a youthful subjective age, and a negative value represents an older subjective age. For example, a score of +0.20 means that a person feels $20 \%$ younger and a score of -0.20 means that an individual feels $20 \%$ older than their actual age. Responses three standard deviations above or below the mean were considered outliers, leading to the exclusion of 16 participants from the analysis.

Individuals also provided information at baseline about their frequency of participation in vigorous (e.g., running or lifting heavy objects) and moderate (e.g., slow or light swimming, brisk walking) leisure physical activity during the summer months and the winter months, which were averaged to give a physical activity score. Baseline BMI was also included, based on self-reported height and weight, and calculated as $\mathrm{kg} / \mathrm{m}^{2}$.

Several covariates were included, such as age (in years), sex (coded as 0 for women and 1 for men), marital status (coded as 1 for married or living with someone and 0 for not married or not living with someone), educational level (using a scale composed of 12 intervals corresponding to sequential educational milestones, from 1 [no grade school] to 12 [doctoral level degree]), and disease burden (measured as the sum of disease and conditions reported by the participants).

\section{Outcome Measures}

Cognitive measures were obtained at the second wave of the MIDUS. In line with prior research, ${ }^{12,34,35}$ two factors were assessed, episodic memory and executive functioning. They were computed on the basis of seven cognitive dimensions measured by the Brief Test of Adult Cognition by Telephone (BTACT). ${ }^{12,34,35}$ This included two measures of episodic memory (immediate and delayed free recall of 15 words), working memory span (backward digit span-the highest span achieved in repeating strings of digits in reverse order), verbal fluency (the number of words produced from the category of animals in 60 seconds), inductive reasoning (completing a pattern in a series of five numbers), processing speed (the number of digits produced by counting backward from 100 in 30 seconds), and attention switching and inhibitory control (the Stop and Go Switch Task). ${ }^{34,35}$ Following exploratory and confirmatory factor analysis, $^{12}$ two cognitive factors were computedepisodic memory (immediate and delayed word recall) and executive functioning (all other measures), by averaging the variables loading on each factor and standardizing to $\mathrm{z}$ scores. ${ }^{12}$ A prior validation study revealed the equivalence of phone testing and faceto-face testing for the BTACT, and shows that the tests used have acceptable psychometric properties in samples who vary widely in age, education, and socioeconomic background. ${ }^{34,35}$ Telephone testing offers the opportunity to reach a range of individuals 
who vary in health status, physical mobility, educational attainment, including those who cannot be tested in person. In addition, the BTACT extended the range of cognitive domains assessed beyond those who are traditionally included in cognitive aging research. ${ }^{34,35}$

\section{Data Analysis}

Separate multiple regressions were used to test whether subjective age predicts episodic memory score or the executive functioning score at follow-up 10 years later. The analyses control for age, sex, marital status, education, disease burden in a first step, frequency of physical activity, and BMI in a second step, and subjective age was included in the third step. We ran collinearity diagnostics through the inspection of tolerance and variance inflation errors, which revealed no problem of multicollinearity. Age, sex, education, marital status, physical activity, disease burden, and BMI were also tested as moderator variables of the association between subjective age and cognitive functioning. A bootstrapping method with $\mathrm{N}=5,000$ bootstrap resample was used for testing the mediating effect of physical activity and BMI on the association between subjective age and both episodic memory and executive functioning, with adjustment for covariates. As a mediation technique, bootstrapping involves resampling the data a preset number of times, in this case 5,000 samples, and estimating the indirect effect in each resample to obtain an empirical approximation of the sampling distribution of the indirect effect along with confidence intervals. ${ }^{36}$ An empirical approximation of the sampling distribution of indirect effects is generated and used to construct $95 \%$ confidence intervals (CI) for the indirect effects. Point estimates of indirect effects are considered significant when zero is not contained in $95 \%$ Cis. $^{36}$

\section{RESULTS}

Baseline characteristics of participants are presented in Table 1. On average, participants reported feeling 19\% younger than their chronological age. For example, the average 60 year old felt as being roughly 10 years younger, an effect similar to what was reported in prior research. ${ }^{17}$ The correlation

\begin{tabular}{lrc}
\hline TABLE 1. Baseline Characteristics of Participants (N $=\mathbf{1 , 3 5 2})$ \\
\hline Variables & M/\% & SD \\
\hline Sex (\% female) & $55 \%$ & - \\
Marital status (\% married/living with someone) & $76 \%$ & - \\
Age (years) & 59.32 & 6.72 \\
Education & 6.79 & 2.53 \\
Disease burden & 2.53 & 2.30 \\
BMI & 27.04 & 4.82 \\
Physical activity & 4.52 & 1.25 \\
Subjective age $\mathrm{a}^{\mathrm{a}}$ & 0.19 & 0.13
\end{tabular}

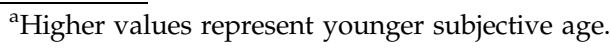

matrix indicated that the subjective age score was unrelated to sex or marital status, but feeling younger, as compared to their age, was more typical of older adults, those with higher education, lower disease burden, higher physical activity level, and lower BMI at baseline (Table 2). Of most interest in this study, a younger subjective age at baseline was correlated with better performance on both episodic memory and executive functioning at follow-up.

As reported in Table 3, regression analysis indicated that chronological age, sex, education, and BMI were significantly associated with episodic memory. Even after accounting for these baseline covariates, subjective age was a significant predictor of episodic memory 10 years later. Consistent with our expectations, subjective age was also positively related to executive functioning, above and beyond the significant association of chronological age, sex, education, and physical activity (see Table 3). For every one standard deviation difference in subjective age score, subjects performed roughly $0.05 \mathrm{SD}$ better in episodic memory and executive functioning. This effect size was roughly similar in magnitude to the effect we found for BMI and physical activity, larger than what we found for disease burden, but decisively smaller than chronological age or educational level.

Additional analysis revealed a significant subjective age by chronological age interaction $(\beta=-0.05$, $\mathrm{t}(1342)=-1.98, \mathrm{p}<0.05)$ for episodic memory, with a younger subjective age associated with better episodic memory more strongly among the younger individuals. The association of subjective age with episodic memory and executive functioning was not moderated by sex, education, marital status, physical activity, disease burden, or BMI.

Bootstrap analysis revealed that the association of subjective age with episodic memory was partially 
TABLE 2. Correlations Between the Variables under Study $(N=1,352)$

\begin{tabular}{|c|c|c|c|c|c|c|c|c|c|}
\hline Variables & 1. & 2. & 3. & 4. & 5. & 6. & 7. & 8. & 9. \\
\hline 1. Sex & - & & & & & & & & \\
\hline 2. Marital status & $0.20^{\text {**:* }}$ & - & & & & & & & \\
\hline 3. Age & -0.01 & -0.04 & - & & & & & & \\
\hline 4. Education & $0.16^{* * \cdots *}$ & 0.01 & $-0.08^{* *}$ & - & & & & & \\
\hline 5. Disease burden & $-0.11^{\text {***** }}$ & $-0.09^{* *}$ & 0.04 & $-0.12^{* * *}$ & - & & & & \\
\hline 6. BMI & $0.09^{* * *}$ & 0.01 & $-0.07^{*}$ & $-0.09^{* * *}$ & $0.18^{* * * *}$ & - & & & \\
\hline 7. Physical activity & $0.25^{* * * *}$ & $0.14^{* * * *}$ & $-0.13^{* * *}$ & $0.18^{* * * *}$ & $-0.20^{* * *}$ & $-0.12^{* * *}$ & - & & \\
\hline 8. Subjective age $^{\mathrm{a}}$ & -0.02 & -0.03 & $0.06^{*}$ & $0.06^{*}$ & $-0.13^{* * * *}$ & $-0.16^{* * * *}$ & $0.07^{\text {*** }}$ & - & \\
\hline 9. Episodic memory & $-0.24^{* * * *}$ & 0.02 & $-0.30^{* * * *}$ & $0.16^{* * * *}$ & -0.01 & $-0.10^{* * * *}$ & $0.06^{*}$ & $0.06^{*}$ & - \\
\hline 10. Executive functioning & $0.15^{* * * *}$ & $0.07^{* *}$ & $-0.31^{* * * *}$ & $0.37^{* * * *}$ & $-0.12^{* * *}$ & -0.04 & $0.18^{* * * *}$ & $0.06^{*}$ & $0.36^{* * * *}$ \\
\hline
\end{tabular}

mediated by BMI, but not by physical activity (Table 4). BMI explained $15 \%$ of the association between subjective age and episodic memory. Thus, those with a younger subjective age performed better on episodic memory tasks in part because they had lower BMIs. The association between subjective age and executive functioning was partially mediated by physical activity, but not by BMI (Table 4). Further analysis of the proportion of the indirect effect revealed that $5 \%$ of the association between subjective age and executive functioning was mediated by physical activity. Thus, those with a younger subjective age performed better on executive functioning task in part through more frequent physical activity.

\section{DISCUSSION}

In a large sample with a prospective design, this study tested and found support for the hypothesis that a younger subjective age is associated with better cognitive functioning 10 years later, even after accounting for chronological age, sex, education, selfreported physical activity, BMI, and disease burden. Therefore, the subjective experience of aging, as indexed by how old or young an individual feels, significantly contributes to the prediction of cognitive performance beyond well-established risk factors for cognitive decline. The effect of subjective age is unlikely to be restricted to one cognitive area, given that we found similar associations with the episodic memory and executive functioning domains. Although relatively small, the strength of the association between subjective age and cognition was comparable or larger than the effects we observed for BMI, disease burden, and physical activity, which are important predictors of cognition in old age. ${ }^{26,28}$

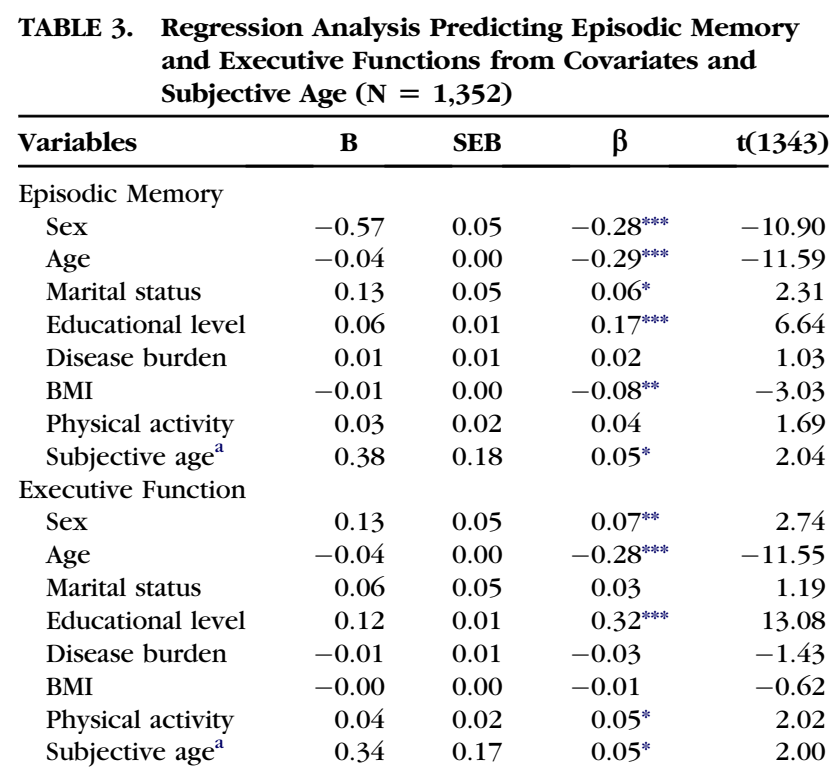

Notes: Model with Episodic Memory as dependent variable: Adjusted $R^{2}=0.193, F_{(8,1343)}=41.30, \mathrm{p}<0.001$; Step 1 (Sex, Age, Marital Status, Educational level, Disease Burden): Adjusted $R^{2}=$ 0.182 , Step 2 (BMI, physical activity): $\Delta R^{2}=0.009, \mathrm{p}<0.001$, Step 3 (Subjective age): $\Delta R^{2}=0.002, \mathrm{p}<0.05$; Model with Executive Function as dependent variable: Adjusted $R^{2}=0.235, F_{(8,1343)}=$ 52.90, p <0.001, Step 1 (Sex, Age, Marital Status, Educational level, Disease Burden): Adjusted $R^{2}=0.231$, Step 2 (BMI, physical activity): $\Delta R^{2}=0.003, \mathrm{p}=0.05$, Step 3 (Subjective age): $\Delta R^{2}=$ $0.002, \mathrm{p}<0.05$.

${ }^{\mathrm{a}}$ Higher values represent younger subjective age. Sex: $0=$ female, $1=$ male; Marital status: $1=$ married/living with someone, $0=$ not married $/$ not living with someone.

${ }^{*} \mathrm{p}<0.05,{ }^{* *} \mathrm{p}<0.01,{ }^{* * *} \mathrm{p}<0.001$. 
TABLE 4. Results from Bootstrap Analysis

\begin{tabular}{|c|c|c|c|c|c|c|}
\hline $\begin{array}{l}\text { Dependent } \\
\text { Variable (DV) }\end{array}$ & $\begin{array}{c}\text { Mediating } \\
\text { Variable (MV) }\end{array}$ & $\begin{array}{c}\text { Effect of IV } \\
\text { on } M^{\mathrm{a}}\end{array}$ & $\begin{array}{l}\text { Effect of MV } \\
\text { on } \mathrm{DV}^{\mathrm{a}}\end{array}$ & $\begin{array}{l}\text { Direct Effect of } \\
\text { Subjective age }\end{array}$ & $\begin{array}{l}\text { Indirect Effect of } \\
\text { Subjective Age }\end{array}$ & $\begin{array}{l}\text { Total Effect of } \\
\text { Subjective age }\end{array}$ \\
\hline \multirow{3}{*}{ Episodic Memory } & & & & $0.38^{*}$ & & $0.47^{*}$ \\
\hline & BMI & $-4.50^{* * * *}$ & $-0.016^{* * *}$ & & $0.07(0.024 ; 0.144)$ & \\
\hline & Physical activity & $0.598^{*}$ & 0.035 & & $0.02(-0.000 ; 0.067)$ & \\
\hline \multirow[t]{3}{*}{ Executive Function } & & & & $0.34^{*}$ & & $0.38^{*}$ \\
\hline & BMI & $-4.50^{* * *}$ & -0.003 & & $0.01(-0.025 ; 0.062)$ & \\
\hline & Physical activity & $0.598^{*}$ & $0.039^{*}$ & & $0.02(0.001 ; 0.070)$ & \\
\hline
\end{tabular}

\footnotetext{
Notes: Total effect of subjective age is calculated without adjustment for physical activity and BMI. Direct effect of physical activity and BMI is calculated with adjustment for subjective age. Direct effect of subjective age is calculated with adjustment for physical activity and BMI. All models are further adjusted for age, sex, marital status, education, and disease burden. DV: dependent variable; IV: independent variable; MV: mediating variable.

${ }^{\mathrm{a} C}$ Coefficients are unstandardized path coefficients, Bootstrap: ${ }^{*} \mathrm{p}<0.05,{ }^{* *} \mathrm{p}<0.01,{ }^{* * *} \mathrm{p}<0.001$.

${ }^{b}$ Bootstrap estimates and Bias Corrected Confidence Interval (CI) for indirect effects of subjective age on episodic memory and executive function through physical activity and BMI.
}

The results revealed that the behavioral pattern and health profile associated with a younger subjective age explain in part its association with cognitive indicators. Feeling younger than one's actual age contributes positively to episodic memory, partially through a lower likelihood of being overweight or obese, and to executive functioning in part through participation in regular physical activity. Furthermore, these findings are consistent with the idea that individuals harboring a younger subjective age are healthier and have more active lifestyles. ${ }^{24,25}$ These individuals may hold resources that allow them to cope with the aging process and to deal with everyday tasks and developmental goals. ${ }^{18}$

The mediators identified in the present study explain only part of the subjective age-cognition relationship, and other factors need to be considered. For example, the higher memory self-efficacy of individuals with a younger subjective age ${ }^{18}$ may lead them to engage and persist in intellectually stimulating activities. In addition, a younger subjective age is considered a self-protective response that is displayed by older adults in response to exposure to negative age stereotypes, ${ }^{37,38}$ and that is also motivated by personality traits such as openness to experience and extraversion, in response to personality stereotypes of aging. ${ }^{39}$ Therefore, feeling younger than one's actual age protects individuals from the influence of negative aging stereotypes, ${ }^{16,33}$ which are recognized as having a consistent deleterious influence on older people's cognitive performance. ${ }^{40}$ Furthermore, given that the American culture puts a strong emphasis on youthfulness, ${ }^{21}$ individuals harboring a younger subjective age may behave more like the typical young individual. Individuals with a younger self-image are more likely to maintain a socially, intellectually, and physically active lifestyle, which in turn is beneficial for their cognitive functioning.

The finding of an association between subjective age and cognitive functioning should be considered as preliminary and needs replication in independent samples. On the other hand, this study is consistent with and extends prior research on the potential of alternative ways of measuring developmental time to explain older adults' cognition. ${ }^{8,9}$ In addition, this study contributes to a growing body of research on the psychological and health-related implications of subjective age, given that it is the first to identify its association with cognitive outcomes.

From a clinical standpoint, the present study suggests that subjective age assessment may inform about individuals at increased risk for potential cognitive deficits in old age. More specifically, individuals who feel older than their age warrant particular attention and may require closer monitoring. Although the effect we observed were relatively small, future studies should test whether an older subjective age may be in itself an early marker of cognitive deficits leading to dementia. Subjective age may also be associated with behavior and outcomes that may amplify cognitive decline. Furthermore, it could be interesting to test whether those who feel older benefit from early intervention or prevention efforts aimed at alleviating cognitive decrements, such as cognitive training programs. In addition, subjective age is modifiable. ${ }^{19}$ For example, 
recent research has found that positive feedback and support directed toward older adults leads to the development of a younger subjective age, which is beneficial for their functioning. ${ }^{19}$ In addition, behavioral interventions may promote a sense of feeling younger. For example, physical activity programs could result in a younger subjective age or mitigate an older subjective age through their influence on positive affect and self-efficacy.

Despite its strength, the current study has several limitations that should be considered before drawing strong conclusions about the role of subjective age. The generalizability of our findings is limited to some extent by the positive selection of the longitudinal participants in the MIDUS. ${ }^{41,42}$ More precisely, the contribution of subjective age observed in the present study may underestimate the true contribution given that participants who dropped out were feeling older, and thus could be considered as being at greater risk of worse cognitive functioning. In addition, the lack of a baseline cognitive assessment in the MIDUS did not allow for testing to determine whether subjective age is associated with changes in cognition over time. The results of the current study, however, indicate that it is worthwhile to further test subjective age as a predictor of cognitive decline. Future studies should also examine whether subjective age can predict changes associated with dementia or other neuropathologies. It is possible that in rating their subjective age, even subclinical dysfunctions unlikely to be diagnosed can be perceived by individuals and incorporated into their self-assessment. It is possible that such ratings predict performance on cognitive tasks because subjective age may be sensitive to nonpathological physiological aging beyond what is accounted by chronological age. Experimental studies are also needed to test whether the manipulation of subjective age can influence one's cognitive performance over long periods.
Although several covariates were included, the present study did not control for other recognized correlates of cognitive functioning in old age, such as frequency of cognitive activity, social networks, perceived health, depressive symptoms, prescription medications, and vascular risk factors. Thus, additional research that accounts for these covariates is needed to test the robustness of the relationship between subjective age and cognition. Furthermore, although comparable to other recognized factors, the size of the direct contribution of subjective age at baseline on later cognition is relatively small. This finding is consistent with prior research which suggests that the age individuals feel is a distal predictor of consequential outcomes and exerts its influence through different pathways. ${ }^{18}$ Therefore, combined with the findings of partial mediation by BMI and physical activity in the present study, one likely possibility is that subjective age drives processes having a more proximal influence on cognitive functioning. Future research is needed to explore other potential behavioral (e.g., involvement in social and cognitive activities), psychosocial (e.g., memory self-efficacy), and physiological pathways (e.g., stress reactivity). The relationships between subjective age, physical activity, and BMI were assessed cross-sectionally. Although the association between subjective age and both BMI and physical activity is theoretically supported, it is also plausible that BMI and physical activity may lead to a younger subjective age.

Despite these limitations, this study reveals that feeling younger than one's actual age may be a protective factor for long-term cognition in old age. Therefore, it paves the way for future research interested in identifying the mechanisms that operate along the age continuum to drive age-related changes in cognitive functioning.

The authors have no disclosures to report.

\section{References}

1. Grigsby J, Kaye K, Baxter J, et al: Executive cognitive abilities and functional status among community-dwelling older persons in the San Luis Valley Health and Aging Study. J Am Geriatr Soc 1998; 46:590-596

2. Yogev-Seligmann G, Hausdorff JM, Giladi N: The role of executive function and attention in gait. Mov Disord 2008; 23:329-342
3. Davis JC, Marra CA, Najafzadeh M, et al: The independent contribution of executive functions to health related quality of life in older women. BMC Geriatr 2010; 10:16

4. Aggarwal NT, Wilson RS, Beck TL, et al: Mild cognitive impairment in different functional domains and incident Alzheimer's disease. J Neurol Neurosurg Psychiatry 2005; 76:1479-1484 
5. Palmer K, Bäckman L, Winblad B, et al: Mild cognitive impairment in the general population: occurrence and progression to Alzheimer disease. Am J Geriatr Psychiatry 2008; 16 : 603-611

6. Singh-Manoux A, Kivimaki M, Glymour MM, et al: Timing of onset of cognitive decline: results from Whitehall II prospective cohort study. BMJ 2012; 344:d7622

7. Daviglus ML, Bell CC, Berrettini W, et al: National Institutes of Health State-of-the-Science Conference statement: preventing Alzheimer disease and cognitive decline. Ann Intern Med 2010; 153:176-181

8. MacDonald SW, Dixon RA, Cohen AL, et al: Biological age and 12year cognitive change in older adults: findings from the Victoria Longitudinal Study. Gerontology 2004; 50:64-81

9. MacDonald SWS, DeCarlo CA, Dixon RA: Linking biological and cognitive aging: towards improving characterizations of developmental time. J Gerontol B Psychol Sci Soc Sci 2011; 66(S1): 59-70

10. Chapman B, Duberstein P, Tindle HA, et al: Personality predicts cognitive function over 7 years in older persons. Am J Geriatr Psychiatry 2012; 20:612-621

11. Cheng ST, Chow PK, Song YQ, et al: Mental and physical activities delay cognitive decline in older persons with dementia. Am J Geriatr Psychiatry 2014; 22:63-74

12. Lachman ME, Agrigoroaei $\mathrm{S}$, Murphy $\mathrm{C}$, et al: Frequent cognitive activity compensates for education differences in episodic memory. Am J Geriatr Psychiatry 2010; 18:4-10

13. Potvin O, Bergua V, Meillon C, et al: State anxiety and cognitive functioning in older adults. Am J Geriatr Psychiatry 2013; 21: 915-924

14. Peters ME, Rosenberg PB, Steinberg M, et al: Neuropsychiatric symptoms as risk factors for progression from CIND to dementia: the Cache County Study. Am J Geriatr Psychiatry 2013; 21 : 1116-1124

15. Montepare JM: Subjective age: Toward a guiding lifespan framework. Int J Behav Dev 2009; 33:42-46

16. Mock SE, Eibach RP: Age prejudice moderates the effect of subjective age on psychological well-being: Evidence from a 10year longitudinal study. Psychol Aging 2011; 26:979-986

17. Rubin DC, Berntsen D: People over forty feel $20 \%$ younger than their age: subjective age across the life span. Psychon Bull Rev 2006; 13:776-780

18. Stephan Y, Caudroit J, Chalabaev A: Subjective health and memory self-efficacy as mediators in the relation between subjective age and life satisfaction among older adults. Aging Ment Health 2011; 15:428-437

19. Stephan Y, Chalabaev A, Kotter-Gruhn D, et al: "Feeling Younger, Being Stronger": an experimental study of subjective age and physical functioning among older adults. J Gerontol B Psychol Sci Soc Sci $2013 ; 68: 1-7$

20. Kotter-Grühn D, Kleinspehn-Ammerlahn A, Gerstorf D, et al: Selfperceptions of aging predict mortality and change with approaching death: 16-year longitudinal results from the Berlin Aging Study. Psychol Aging 2009; 24:654-667

21. Westerhof GJ, Barrett AE: Age identity and subjective well-being: a comparison of the United States and Germany. J Gerontol B Psychol Sci Soc Sci 2005; 60B:S129-S136

22. Schafer MH, Shippee TP: Age identity, gender, and perceptions of decline: does feeling older leads to pessimistic dispositions about cognitive aging? J Gerontol B Psychol Sci Soc Sci 2010; 65B: S91-S96
23. Valentijn SAM, Hill RD, Van Hooren SAH, et al: Memory selfefficacy predicts memory performance: Results from a 6-year follow-up study. Psychol Aging 2006; 21:165-172

24. Demakakos P, Gjonca E, Nazroo J: Age identity, age perceptions, and health: evidence from the English Longitudinal Study of Ageing. Ann NY Acad Sci 2007; 1114:279-287

25. Caudroit J, Stephan Y, Chalabaev A, et al: Subjective age and social cognitive determinants of physical activity among active older adults. J Aging Phys Act 2012; 20:484-496

26. Weuve J, Kang JH, Manson JE, et al: Physical activity, including walking, and cognitive function in older women. JAMA 2004; 292:1454-1461

27. Kramer AF, Erickson KI, Colcombe J: Exercise, cognition, and the aging brain. J Appl Physiol 2006; 101:1237-1242

28. Singh-Manoux A, Czernichow S, Elbaz A, et al: Obesity phenotypes in midlife and cognition in early old age: The Whitehall II cohort study. Neurology 2012; 79:755-762

29. Brim OG, Ryff CD, Kessler RC: The MIDUS National Survey: an overview. In How Healthy Are We? A National Study of Well-being at Midlife. Edited by Brim OG, Ryff CD, Kessler RC. Chicago, IL, University of Chicago Press, 2004, pp 1-34

30. Brim OG, Baltes PB, Bumpass LL, et al: National Survey of Midlife Development in the United States (MIDUS), 1995-1996. Boston, MA Harvard Medical School, Department of Health Care Policy, 1996

31. Radler BT, Ryff CD: Who participates? Accounting for longitudinal retention in the MIDUS National Study of Health and Wellbeing. J Aging Health 2010; 22:307-331

32. Kleinspehn-Ammerlahn A, Kotter-Grühn D, Smith J: Self-perceptions of aging: do subjective age and satisfaction with aging change during old age? J Gerontol B Psychol Sci Soc Sci 2008; 63B:377-385

33. Eibach RP, Mock SE, Courtney EA: Having a "senior moment": induced aging phenomenology, subjective age, and susceptibility to ageist stereotypes. J Exp Soc Psychol 2010; 46:643-649

34. Tun PA, Lachman ME: Age differences in reaction time and attention in a national telephone sample of adults: education, sex, and task complexity matter. Dev Psychol 2008; 44:1421-1429

35. Tun PA, Lachman ME: Telephone assessment of cognitive function in adulthood: the Brief Test of Adult Cognition by Telephone (BTACT). Age Ageing 2006; 35:629-632

36. Preacher KJ, Hayes AF: Asymptotic and resampling strategies for assessing and comparing indirect effects in multiple mediator models. Behav Res Methods 2008; 40:879-891

37. Weiss D, Freund AM: Still young at heart: negative age-related information motivates distancing from same-aged people. Psychol Aging 2012; 27:173-180

38. Weiss D, Lang FR: "They" are old but "I" feel younger: Age-group dissociation as a self-protective strategy in old age. Psychol Aging 2012; 27:153-163

39. Stephan Y, Demulier V, Terracciano A: Personality, self-rated health and subjective age in a life-span sample: the moderating role of chronological age. Psychol Aging 2012; 27:875-880

40. Levy BR: Stereotype embodiment: a psychosocial approach to aging. Curr Dir Psychol Sci 2009; 18:332-336

41. Turiano NA, Pitzer L, Armour C, et al: Personality trait level and change as predictors of health outcomes: findings from a national study of Americans (MIDUS). J Gerontol B Psychol Sci Soc Sci 2012; 67:4-12

42. Graham EK, Lachman ME: Personality stability is associated with better cognitive performance in adulthood: are the stable more able? J Gerontol B Psychol Sci Soc Sci 2012; 67:545-554 